\title{
Comprehensive Influences of Overexpression of a MYB Transcriptor Regulating Anthocyanin Biosynthesis on Transcriptome and Metabolome of Tobacco Leaves
}

\author{
Yuan Zong ${ }^{1,2,+}$, Shiming $\mathrm{Li}^{1,2,+}$, Xinyuan $\mathrm{Xi}^{1,2,3}$, Dong Cao ${ }^{1,2,3}$, Zhong Wang ${ }^{4}$, Ran Wang ${ }^{4, *}$ \\ and Baolong Liu 1,3,4,* \\ 1 Key Laboratory of Adaptation and Evolution of Plateau Biota (AEPB), Northwest Institute of Plateau \\ Biology, The Innovative Academy of Seed Design, Chinese Academy of Sciences, Xining 810008, China; \\ zongyuan@nwipb.cas.cn (Y.Z.); lishimingbgi@163.com (S.L.); xixy@nwipb.cas.cn (X.X.); \\ caodong@nwipb.cas.cn (D.C.) \\ 2 Qinghai Province Key Laboratory of Crop Molecular Breeding, Xining 810008, China \\ 3 University of Chinese Academy of Sciences, Beijing 100049, China \\ 4 National Tobacco Research Center, Zhengzhou Tabacco Research Institute, Zhengzhou 450001, China; \\ 13812552555@163.com \\ * Correspondence: wangr@ztri.com.cn (R.W.); blliu@nwipb.cas.cn (B.L.) \\ + Yuan Zong and Shiming Li are co-first authors.
}

Received: 21 September 2019; Accepted: 14 October 2019; Published: 16 October 2019

check for updates

\begin{abstract}
Overexpression of R2R3-MYB transcriptor can induce up-expression of anthocyanin biosynthesis structural genes, and improve the anthocyanin content in plant tissues, but it is not clear whether the MYB transcription factor overexpression does effect on other genes transcript and chemical compounds accumulation. In this manuscript, RNA-sequencing and the stepwise multiple ion monitoring-enhanced product ions (stepwise MIM-EPI) strategy were employed to evaluate the comprehensive effect of the MYB transcription factor LrAN2 in tobacco. Overexpression of LrAN2 could promote anthocyanin accumulation in a lot of tissues of tobacco cultivar Samsun. Only 185 unigenes express differently in a total of 160,965 unigenes in leaves, and 224 chemical compounds were differently accumulated. Three anthocyanins, apigeninidin chloride, pelargonidin 3-O-beta-D-glucoside and cyanidin 3,5-O-diglucoside, were detected only in transgenic lines, which could explain the phenotype of purple leaves. Except for anthocyanins, the phenylpropanoid, polyphenol (catechin), flavonoid, flavone and flavonol, belong to the same subgroups of flavonoids biosynthesis pathway with anthocyanin and were also up-accumulated. Overexpression of LrAN2 activated the bHLH (basic helix-loop-helix protein) transcription factor $A N 1 b$, relative to anthocyanin biosynthesis and the MYB transcription factor $M Y B 3$, relative to proanthocyanin biosynthesis. Then, the structural genes, relative to the phenylpropanoid pathway, were activated, which led to the up-accumulation of phenylpropanoid, polyphenol (catechin), flavonoid, flavone, flavonol and anthocyanin. The MYB transcription factor $C P C$, negative to anthocyanin biosynthesis, also induced up-expression in transgenic lines, which implied that a negative regulation mechanism existed in the anthocyanin biosynthesis pathway. The relative contents of all 19 differently accumulated amino and derivers were decreased in transgenic lines, which meant the phenylalanine biosynthesis pathway completed the same substrates with other amino acids. Interestingly, the acetylalkylglycerol acetylhydrolase was down-expressed in transgenic lines, which caused 19 lyso-phosphatidylcholine and derivatives of lipids to be up-accumulated, and 8 octodecane and derivatives were down-accumulated. This research will give more information about the function of MYB transcription factors on the anthocyanin biosynthesis and other chemical compounds and be of benefit to obtaining new plant cultivars with high anthocyanin content by biotechnology.
\end{abstract}


Keywords: MYB transcriptor; secondary metabolism; anthocyanin; transcriptome; metabolome

\section{Introduction}

Anthocyanins are glycosylated polyphenolic compounds, and endow flowers, seeds, fruits, and vegetative tissues with a range of colors varying from orange, red, and purple to blue [1]. The intravacuolar environment can influence the color phenotype of anthocyanins because of the characteristics of water-soluble and cell vacuoles location [2]. Over 600 anthocyanins have been identified in nature. The most common anthocyanins are the derivatives of six widespread anthocyanidins, namely, pelargonidin, cyanidin, delphinidin, peonidin, petunidin, and malvidin. Anthocyanins can protect plants against various biotic and abiotic stresses, partially due to their powerful antioxidant properties. In addition, anthocyanin-rich food products have become increasingly popular due to their attractive colors and suggested benefits for human health [3].

Anthocyanins belong to a diverse family of aromatic molecules called flavonoids. Flavonoids contain five major subgroups beside anthocyanins in higher plants: chalcones, flavones, flavonols, flavandiols or proanthocyanidins, and aurones. Most of the structural genes in the anthocyanin biosynthesis pathway have been identified in various plants, such as apple (Malus domestica), Arabidopsis (Arabidopsis thaliana), grapevine (Vitis vinifera L.), and petunia (Petunia hybrida) [4-7]. It is known that the structural genes of anthocyanin biosynthesis contain phenylalanine ammonia lyase (PAL), cinnamate 4-hydroxylase (C4H), 4-coumarate-CoA ligase (4CL), chalcone synthase (CHS), chalcone isomerase $(C H I)$, flavanone 3-hydroxylase $(F 3 H)$, flavonoid 3-hydroxylase $\left(F 3^{\prime} H\right)$ or flavonoid $3^{\prime}, 5^{\prime}$-hydroxylase $\left(F 3^{\prime} 5^{\prime} H\right)$, dihydroflavonol 4-reductase (DFR), anthocyanidin synthase (ANS), and O-methyltransferase (OMT) [8]. The transcription of the structural genes is regulated directly by the MYB-bHLH-WD40 complex (MBW), which is composed of R2R3-MYB, bHLH, and WD40 proteins [9]. the transcriptional activation of MYB transcription factors can usually lead to anthocyanin accumulation in the leaves or fruits in plants [10]. In apples, red fruit color is associated with MdMYB10 [11], while the purple phenotype of sweet potato is controlled by IbMYB1 [12]. LhMYB6 and LhMYB12 can activate the transcription of the anthocyanin biosynthetic structural genes and cause anthocyanin accumulation in petunia (Petunia hybrida) [13].

The overexpression of only MYB transcription factors has been used to enhance anthocyanin content in several plant species. Pattanaik reported that overexpression of the MYB transcription factor $N t A n 2$ enhanced expression levels of the anthocyanin biosynthetic structural genes and anthocyanin accumulation in tobacco [14], whereas the overexpression of PtrMYB119 also do the same things in a number of tissues of hybrid poplar [15]. Although it has been proven that the overexpression of MYB transcription factors promotes anthocyanin accumulation in plants, the molecular mechanism has not been clarified in detail. The plant cell is a complex system. The overexpression of a transcription factor will activate structural genes and the expression of structural genes will produce some kind of chemical compound [16]. The plant cell will adapt to changes in the accumulation of chemical compounds within it. Previous researchers have reported on the transcription of a limited number of genes, in response to overexpression of MYB transcription factors, and the accumulation of a limited number of chemical compounds $[17,18]$. These reports have not clarified the number of genes affected by the overexpression of MYB transcription factors and the turbulence effects of MYB transcription factor overexpression on chemical compounds other than anthocyanin.

Recently, the stepwise multiple ion monitoring-enhanced product ions (stepwise MIM-EPI) strategy was designed to analyze widely targeted metabolomes. The method can quantify hundreds of metabolites simultaneously in rice leaf [19]. Moreover, high-throughput RNA-sequencing (RNA-seq) has emerged as a powerful and cost-efficient tool in providing comprehensive information of the nucleotide sequence and transcript profiling of all genes expressed in the specific tissues of plants [20-22]. In this manuscript, these two technologies were employed to uncover the transcript and metabolite 
differences in the leaves of wild-type (WT) and transgenic lines with high anthocyanin content. It is the first time to reveal the comprehensive function of the MYB transcriptor factor on the transcript and metabolite, except for anthocyanin biosynthesis.

\section{Results}

\subsection{Phenotype and Anthocyanin Content of Transgenic Line Overexpressing LrAN2}

As mentioned previously, LrAN2 is a MYB transcription factor expressed only in the black fruit of Lycium ruthenicum Murry [23]. Overexpression of LrAN2 causes visible anthocyanin accumulation in various parts of the plant (Figure 1A). The relative anthocyanin contents of roots, stems, leaves, flowers, and seeds were significantly higher in the transgenic lines than the WT (Figure 1B). Although anthocyanin differences are not visible in roots, the contents of this compound were found to be different in the two lines using chemical measurements. Because the anthocyanin content of the leaves was the highest in the three tested transgenic lines, the leaves were chosen for further transcriptome and metabolome analysis.
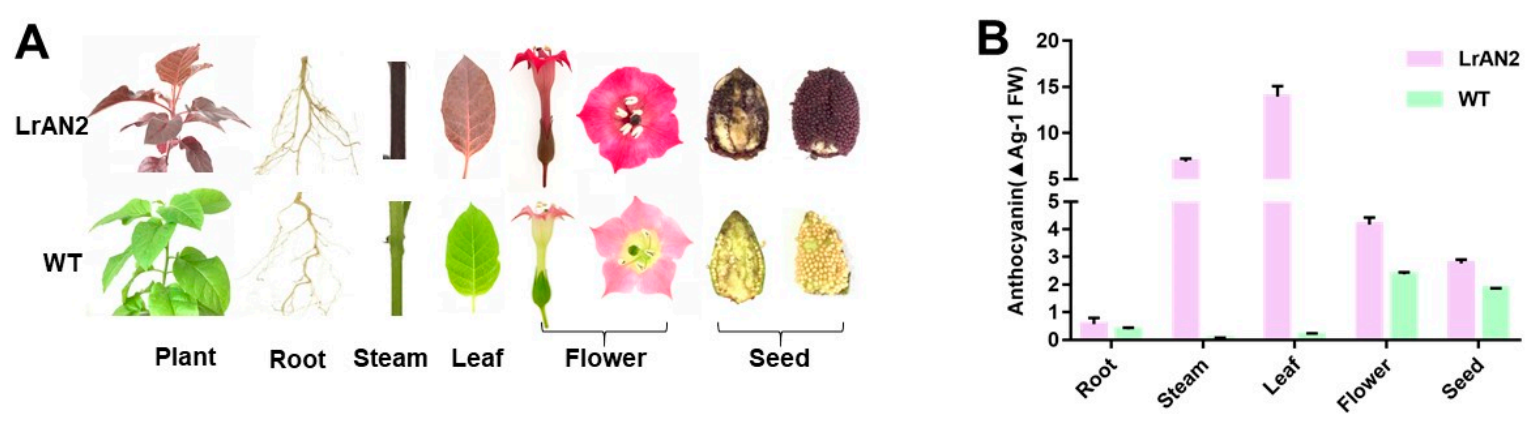

Figure 1. Phenotype and anthocyanin content in transgenic lines and wild-type (WT). (A) The phenotype of roots, stems, leaves, seeds, and flowers of transgenic lines and WT. (B) The anthocyanin content in different tissues of transgenic lines and WT.

\subsection{Chemical Compounds Difference in Transgenic Lines and Wild-Type (WT)}

The stepwise MIM-EPI strategy was developed recently to analyze widely targeted metabolomes. The overlapping display analysis of total ion flow charts (TIC) for LC-MS/MS detection and analysis of different quality control samples showed that the repeatability and reliability of the data were good enough to enable further analysis (Figure S1). A total of 639 chemical compounds from 22 classes were detected and 224 compounds from 19 classes exhibited significantly different levels of accumulation in the transgenic lines and WT (Table 1). Transgenic lines displayed higher contents of 109 chemical compounds and the WT showed increased levels of 115 compounds. Although 13 alcohols, two quinones, and five sterides were found, none of these accumulated differently in transgenic lines and the WT (Table S2).

The average ratio of differently accumulated/total compounds was $35.05 \%$. The ratios of the nine classes were bigger than $35.05 \%$ (Table 1). The first seven classes included anthocyanins, polyphenols, flavonols, flavonoids, flavones, flavanones, and phenylpropanoids. All these classes of chemical compounds belong to the flavonoid biosynthesis pathway. The phenylpropanoids, polyphenols, flavones, flavonols, flavonoids, and anthocyanins showed more upregulation in the transgenic lines, except for the compound flavone (Table 1). A total of seven anthocyanins, peonidin O-hexoside, rosinidin O-hexoside, delphinidin, cyanidin 3,5-O-diglucoside (Cyanin), pelargonidin 3-O-beta-D-glucoside (callistephin chloride), peonidin 3-O-glucoside chloride, and apigeninidin chloride, were identified (Table S2). Seven anthocyanins showed higher levels of accumulation in transgenic lines, while only one accumulated more highly in the WT (Tables 1 and S2). The raised accumulation of anthocyanin may be the reason for purple colored leaves in the transgenic 
lines. Three anthocyanins, including cyanidin 3,5-O-diglucoside, pelargonidin 3-O-beta-D-glucoside, and apigeninidin chloride, were only increased significantly in transgenic lines (Table S2). The remaining three classes included lipids, vitamins and derivatives, and terpene. The class of compounds containing vitamins and their derivatives showed the same levels of up- and down-regulation (Table 1). Among the lipids, 10 compounds were more highly accumulated in transgenic lines, while 21 were reduced (Table S2). Interestingly, 19 compounds showing higher levels of production belonged to the lyso-phosphatidylcholines (and derivatives), while 8 downregulated compounds belonged to the octodecanes (and derivatives, Table S2). Interestingly, all 16 differently accumulated amino acids exhibited lower levels of production in the transgenic lines (Tables 1 and S2).

Table 1. Summary of differently accumulated chemical compounds in the transgenic lines and WT.

\begin{tabular}{|c|c|c|c|c|c|}
\hline Category & $\begin{array}{c}\text { Total } \\
\text { Compounds }\end{array}$ & $\begin{array}{l}\text { Differently } \\
\text { Accumulated } \\
\text { Compounds }\end{array}$ & Percent $(\%)$ & $\begin{array}{l}\text { Up-Accumulation } \\
\text { Compounds }\end{array}$ & $\begin{array}{c}\text { Down- } \\
\text { Accumulation } \\
\text { Compounds }\end{array}$ \\
\hline Polyphenol & 12 & 8 & 66.67 & 7 & 1 \\
\hline Flavonol & 29 & 17 & 58.62 & 12 & 5 \\
\hline Flavonoid & 18 & 10 & 55.56 & 8 & 2 \\
\hline Phenylpropanoids & 53 & 25 & 47.17 & 18 & 7 \\
\hline Lipids & 68 & 31 & 45.59 & 10 & 21 \\
\hline Vitamins and derivatives & 15 & 6 & 40.00 & 3 & 3 \\
\hline Terpene & 17 & 6 & 35.29 & 2 & 4 \\
\hline Organic acids and derivatives & 97 & 34 & 35.05 & 17 & 17 \\
\hline Isoflavone & 6 & 1 & 16.67 & 0 & 1 \\
\hline Carbohydrates & 19 & 3 & 15.79 & 1 & 2 \\
\hline Alkaloids & 33 & 5 & 15.15 & 1 & 4 \\
\hline Indole derivatives & 7 & 1 & 14.29 & 0 & 1 \\
\hline Alcohols & 13 & 0 & 0.00 & 0 & 0 \\
\hline Quinones & 2 & 0 & 0.00 & 0 & 0 \\
\hline Sterides & 5 & 0 & 0.00 & 0 & 0 \\
\hline Total & 639 & 224 & 35.05 & 109 & 115 \\
\hline
\end{tabular}

\subsection{Differently-Expressed Genes in Transgenic Lines and WT}

Using transcriptome sequencing, the total number of bases from all samples was over $6 \mathrm{~Gb}$ after filtering (Table S3). The clean reads were further assembled into 160,965 unigenes. The average length of the unigenes was $1233 \mathrm{bp}$ and the length of N50 was $2002 \mathrm{bp}$ (Table S4). A Blast X search resulted in a total of 135,911 predicted proteins being predicted (Table S5).

Although a total 160,965 unigenes were found, only 185 were expressed differently in the transgenic lines and WT (Figure 2A, Table S6). Compared with the WT, 39 unigenes were downregulated and 146 were upregulated (Figure 2A, Table S6). Fifteen pathways contained only upregulated unigenes in the enriched 30 Kyoto Encyclopedia of Genes and Genomes (KEGG) pathways (Figure 2B). The pathways included amino sugar and nucleotide sugar metabolism, basal transcription factors, cholesterol metabolism, cyanoamino acid metabolism, flavone and flavonol biosynthesis, flavonoid biosynthesis, galactose metabolism, glucosinolate biosynthesis, glutathione metabolism, mitogen-activated protein kinase (MAPK) signaling pathway, nitrogen metabolism, phenylpropanoid biosynthesis, porphyrin and chlorophyll metabolism, starch and sucrose metabolism, and the sulfur relay system. Seven pathways contained only downregulated unigenes. These pathways were lipid metabolism, fatty acid biosynthesis, fatty acid degradation, fatty acid metabolism, monobactam biosynthesis, phagosome assembly, and soluble $\mathrm{N}$-ethylmaleimide-sensitive fusion protein attachment protein receptors (SNARE) interactions in vesicular transport (Figure 2B). 

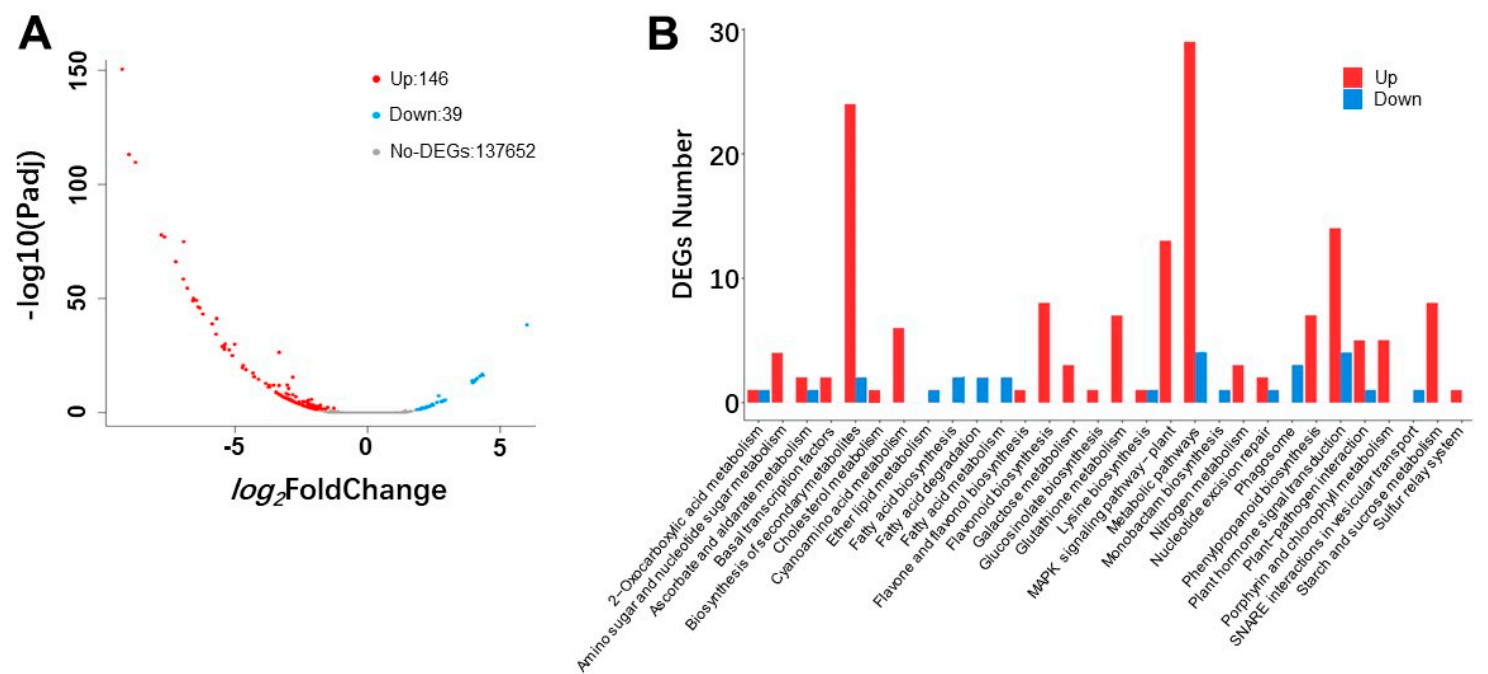

Figure 2. Differentially expressed unigenes (DEGs) in transgenic lines and WT. (A) The volcano distribution map of all DEGs. The $\mathrm{X}$-axis represents the FoldChange in expression after conversion of the $\log 2$ value. The Y-axis represents the value of $-\log 10$ (Padj). The blue point represents the upregulated genes (log2FoldChange $\geq 1$, Padj $\leq 0.05$ ), 146 unigenes expressed in transgenic lines were higher than the control. The red point represents the downregulated genes (log2FoldChange $\leq-1$, Padj $\leq 0.05), 39$ unigenes expressed in transgenic lines were lower than the control. The gray point represents no DEGs. 137,652 unigenes did not express differently in the transgenic lines and WT. (B) Enrichment Pathway Map of DEGs.

The top thirty upregulated unigenes were homologous to the genes related to anthocyanin biosynthesis (Table S6), including ANS, DFR, GST, LrAN2, difF, AN1b, ANP (anthocyanin permease), $M Y B 3$, and so on. Apart from being related directly to anthocyanin biosynthesis, ANP is a multidrug resistance-associated protein that plays an important role in the transport of anthocyanin pigments into vacuoles [24], while difF encodes a cytochrome b5, which is required for full activity of flavonoid $3^{\prime}, 5^{\prime}$-hydroxylase [25]. The log2FoldChange value of the largest upregulated unigene ANS reached 9.26 and this was followed by DFR (9.00; Table S6). The total FPKM of the unigenes homologous to PAL, $C 4 H, 4 C L, C H S, C H I, F 3 H, F 3^{\prime} H, F 3^{\prime} 5^{\prime} H, D F R$, and ANS were compared in transgenic lines and the WT. These structural anthocyanin biosynthesis genes were significantly upregulated, except for $4 C L, F 3 H$, and $F 3^{\prime} 5^{\prime} H$ (Figure 3A, Table S7). The qPCR experiment confirmed the expression differences between these structural genes (Figure 3B). Undoubtedly, the anthocyanin biosynthesis pathway is activated by the overexpression of MYB transcription factor LrAN2. Moreover, 10 upregulated unigenes were homologous to bHLH transcription factor $A N 1 b$, which has been proven to regulate anthocyanin biosynthesis [26] (Table S7). Interestingly, ten and one unigenes homologous to transcription factors MYB3 and CPC respectively, showed more expression in transgenic lines (Tables S6 and S7).

The anthocyanin biosynthetic pathway and its branches were selected separately, and their structural genes and metabolites were analyzed. Biological reactions with (E)-p-coumaric acid as a substrate showed that the expression of these metabolites: Phloretin (Flavanone), Caffeic acid (Phenylpropanoids), Sinapoyl malate (organic acids and derivatives), Myricetin (Flavonol), Cyanidin (Anthocyanins), Pelargonidin (Anthocyanins), in purple tobacco increased at least ten times (measured by $\log 2$ value). The expression of structural genes DFR and ANS in the anthocyanin biosynthesis pathway was the highest among all structural genes, which was consistent with the results of qPCR (Figure 3B). 


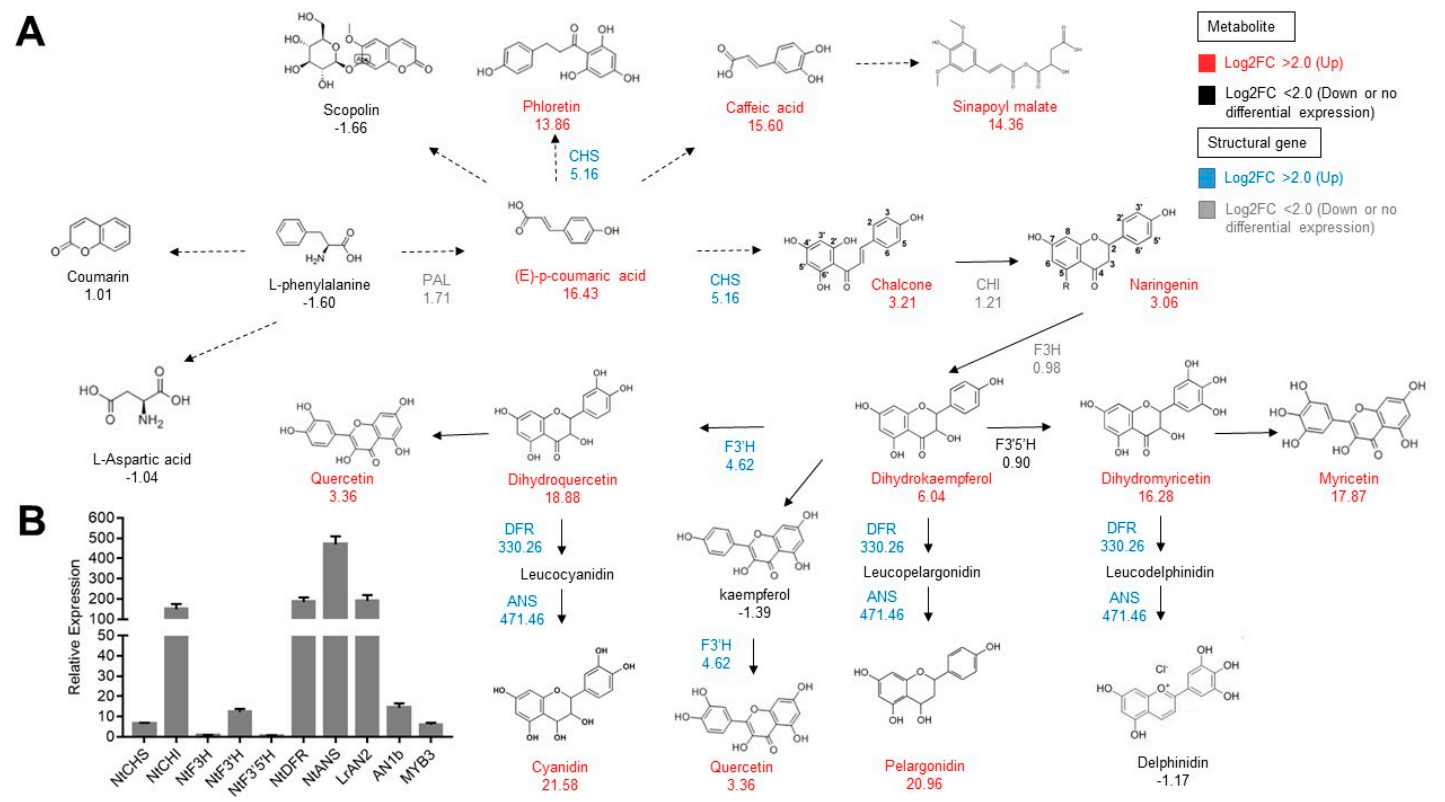

Figure 3. The relative expression levels of the unigenes related to anthocyanin biosynthesis. (A) The biosynthesis pathway. Arrows show the metabolic stream, left or upward arrows represent the genes catalyzing the progress, the blue abbreviations express the genes found in the assembly unigenes, and the number under the genes represents the relative expression level in transgenic lines versus the WT. The numbers under the chemical compounds represent the relative contents of the compounds accumulated in the transgenic lines versus the WT. These structural anthocyanin biosynthesis genes were more significantly upregulated in transgenic lines than WT. (B) The relative transcript level of the genes relative to anthocyanin biosynthesis based on qPCR experiments.

\subsection{Conjoint Analysis of Transcriptome and Metabolome}

To further evaluate the effects of the transcriptome change on the metabolome, the differently-expressed genes and differently-accumulated compounds were put on the KEGG pathway. Fourteen pathways contained both differently expressed genes and differently accumulated compounds (Figure 4, Table S8). These were divided further into three classes: the phenylpropanoid secondary metabolism pathway, the amino acid biosynthesis and metabolism pathway, and the ether lipid metabolism pathway. The phenylpropanoid secondary biosynthesis pathway consisted of flavonoid biosynthesis, phenylpropanoid biosynthesis, and flavone and flavonol biosynthesis, while the amino acid biosynthesis and metabolism pathway consisted of monobactam biosynthesis, the sulfur relay system, 2-oxocarboxylic acid metabolism, amino sugar and nucleotide sugar metabolism, glucosinolate biosynthesis, phenylalanine metabolism, cysteine and methionine metabolism, glycine, serine and threonine metabolism, aminoacyl-tRNA biosynthesis, and biosynthesis of amino acids. Only flavonoid biosynthesis could be enriched based on the $p$-value of the transcriptome and metabolome (Figure 4). In the ether lipid metabolism pathway, acetylalkylglycerol acetylhydrolases showed lower expression in the transgenic lines than WT (Table S8). The acetylalkylglycerol acetylhydrolases may catalyze 1-alkyl-2-acetyl-sn-glycero-3-phosphocholine and $\mathrm{H}_{2} \mathrm{O}$ to produce 1-alkyl-sn-glycero-3-phosphocholine and acetate [27]. 1-alkyl-2-acetyl-sn-glycero-3-phosphocholine is the precursor of lyso-phosphatidylcholine and derivatives, while 1-alkyl-sn-glycero-3-phosphocholine is the precursor of octodecane and its derivatives [27]. The downregulation of the platelet-activating factor acetylhydrolase could explain why 19 lyso-phosphatidylcholines and derivatives were upregulated in transgenic lines and 8 octodecanes and derivatives were produced at lower levels in metabolome analysis. 


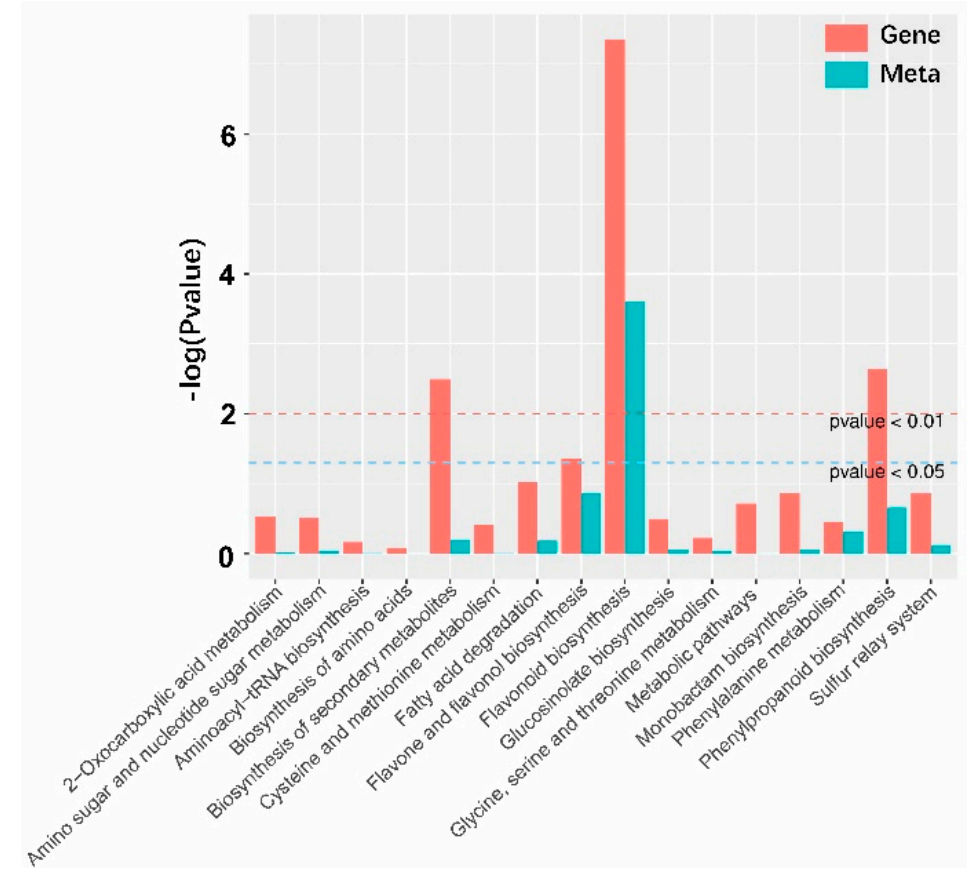

Figure 4. The Kyoto Encyclopedia of Genes and Genomes (KEGG) enrichment histogram of conjoint analysis of differently expressed genes and accumulated metabolites. The X-axis represents the metabolic pathways, the Y-axis represents the expression as -log ( $p$-value). The red columns represent the enrichment $p$-values of differentially expressed genes, and the green columns represent the enrichment $\mathrm{p}$-values of different metabolites.

\section{Discussion}

In this manuscript, we described the effect of LrAN2 overexpression on the transcriptome and metabolome of tobacco leaves using stepwise MIM-EPI and RNA-seq. These were proven to be effective strategies for analyzing the comprehensive metabolome and transcriptome, respectively. A total of 639 chemical compounds from 22 classes was detected and 160,965 unigenes were assembled during transcriptome analysis.

\subsection{Transcriptome Turbulence Was Relatively Simple and Metabolome Influence Was Extensive}

Interestingly, very few genes showed different levels of expression in transgenic lines and WT using transcriptome analysis. Although many unigenes were assembled, only 185 were shown to be expressed differently, which is a very low number. The top 30 unigenes showing upregulation were related to anthocyanin biosynthesis. These unigenes contained direct structural genes such as ANS, $D F R$, and transcription factors related to anthocyanin biosynthesis, but also ANP and difF, which are accessories that have not been identified in many plants. It could be speculated that overexpression of some regulatory factors with RNA-seq analysis could help in the isolation of structural genes and regulation factors related to specific chemical compound biosynthetic pathways. In this case, a lot of differently expressed unigenes were annotated, particularly upregulated unigenes, but the functions of the remaining unigenes are still unknown. Crispr/Cas9 was used to knock out these differently expressed genes to determine their functions [28].

Of 639 chemical compounds, the contents of 224 were significantly different in the transgenic lines and WT. The ratio of differently accumulated compounds to total identified compounds reached $35.05 \%$. Compared to that of the transcriptome, the influence of the metabolome was extensive. 


\subsection{Overexpression of MYB Transcription Factors Influences Phenylpropanoid Metabolism}

In many plants, it has been noted that overexpression of a MYB transcription factor enhances the expression of anthocyanin biosynthetic genes as well as anthocyanin accumulation. This research further confirmed that the chemical classes of phenylpropanoids, polyphenols, flavones, flavonols, flavonoids, and flavanones showed high levels of accumulation (except anthocyanins) in transgenic lines. Moreover, overexpression of LrAN2 could promote the bHLH transcriptor AN1b, which is related to anthocyanin biosynthesis. Transcriptional activation of the MBW complex may regulate the transcription of anthocyanin biosynthetic genes directly. LrAN2 also promotes the transcription of other transcription factors, homologous to $M Y B 3$ and $C P C$. The transcription factor MYB3 is believed to regulate proanthocyanin (epiafzelechin and derivatives) biosynthesis [29], while $C P C$ negatively regulates anthocyanin biosynthesis in Arabidopsis as it competes with the R2R3-MYB transcription factor PAP1/2 [30]. The accumulation of epiafzelechin and derivatives in the transgenic lines could be explained by the up-expression of $M Y B 3$. The upregulation of $C P C$ due to anthocyanin accumulation is a mechanism for reducing anthocyanin biosynthesis.

\subsection{Amino Acid Biosynthesis is Influenced by the Overexpression of MYB Transcription Factor}

Phenylalanine is the substrate for the phenylpropanoid biosynthesis pathway, and it is an amino acid. Undoubtedly, phenylalanine competes for the same substrates as other amino acids. The relative contents of all 16 differently accumulated amino acids and derivatives were decreased in the transgenic lines. In conjoint analysis of the transcriptome and metabolome, apart from the phenylpropanoid secondary biosynthesis pathway and fatty acid degradation pathway, the enriched KEGG pathways exhibiting different gene expression and chemical compound levels were related to amino acid biosynthesis and metabolism. The cyanoamino acid metabolism pathway derived from phenylalanine was active in the transcriptome. Although a chemical difference has not been found, this metabolic pathway is connected directly to those of glycine, serine and threonine, cystine and methionine, glutathione, and other amino acids.

This research describes a relatively comprehensive change in the metabolome and transcriptome of tobacco leaves following overexpression of a MYB transcription factor. Six transgenic tobacco strains overexpressing LrAN2 were obtained. This study provides more information about the function of MYB transcription factors in anthocyanin biosynthesis and that of other chemical compounds. These results will help researchers to establish new plant cultivars containing high levels of anthocyanin through transgenic technology.

\section{Materials and Methods}

\subsection{Materials}

The Nicotiana tabacum L. cultivar Samsun was chosen as a transformation plant. All plant materials were stored in the Northwest Plateau Institute of Biology, Chinese Academy of Sciences. Samsun served as the control group and transgenic tobacco served as the experimental group. Each group was repeated three times. Transcriptome sequencing and metabolome sequencing used the same tobacco leaf. Chemical and molecular regents were purchased from Gansu Pengcheng Biological Technology Development Company.

\subsection{Obtaining Transgenic Lines with Overexpression of MYB Transcription Factor}

The MYB transcription factor LrAN2 was first reported by our laboratory. It expressed only in the black fruit of Lycium ruthenicum Murry and the allele variation of LrAN2 is strictly related to the black fruit trait [23]. In this study, LrAN2 was transformed into tobacco Samsun to obtain transgenic lines with high anthocyanin content. The vector PJAM1502 with a double $35 \mathrm{~s}$ promoter was used for the transformation. The construct PJAM1502:LrAN2 was established using a Gateway Cloning Kit (Invitrogen, Carlsbad, CA, USA). The freeze-melt method was employed to transform the binary 
vectors into Agrobacterium tumefaciens strain GV3101. The leaf disc transformation method was used for the tobacco transformation [31]. Regeneration tissues were grown on selective media $(0.7 \%(\mathrm{w} / \mathrm{v})$ agar, $3 \%(\mathrm{w} / \mathrm{v})$ sucrose, $1.0 \mathrm{mg} / \mathrm{L}$ 1-naphthaleneacetic acid (NAA), $1.0 \mathrm{mg} / \mathrm{L}$ 6-benzylaminopurine (6-BA), and $150 \mathrm{mg} / \mathrm{L}$ kanamycin. The positive shoots were planted in the greenhouse under long-day lighting (16 h light/ $8 \mathrm{~h}$ dark). Though 23 positive lines were obtained, only three T3 family lines carrying the objective gene without segregation of character were selected for further experiments.

\subsection{Anthocyanin Content Measurement}

The "Total Monomeric Anthocyanin Pigment Content of Fruit Juices, Beverages, Natural Colorants, and Wines" (AOAC (Association of Official Analytical Chemists) Official Method 2005.02) method was used to measure relative anthocyanin content. $\mathrm{HCl}(1 \% \mathrm{v} / \mathrm{v})$ was added to $100 \mathrm{mg}$ of comminuted plant tissues (root, steam, leaf, seed, and flower), and the mixtures were incubated at $4{ }^{\circ} \mathrm{C}$ overnight in the dark to extract anthocyanin, with three repetitions in each plant.

\subsection{Analysis of Chemical Contents}

Transgenic lines and WT were cultured in a plant incubator for 1 month. The leaves were stripped and stored in a refrigerator at $-80{ }^{\circ} \mathrm{C}$ before chemical and transcriptome analyses. Freeze-dried leaves were crushed with a mixer mill (MM 400, Retsch) at $30 \mathrm{~Hz}$ with a zirconia bead for $1.5 \mathrm{~min}$. $100 \mathrm{mg}$ powder was weighed and extracted overnight with $1.0 \mathrm{~mL} 70 \%$ aqueous methanol at $4{ }^{\circ} \mathrm{C}$. After centrifugation at $10,000 \times g$ for $10 \mathrm{~min}$, the extracts were filtered by the $0.22 \mu \mathrm{m}$ filter membrane (ANPEL, Shanghai, China, http://www.anpel.com.cn/).

An LC-electrospray ionization-tandem MS (ESI MS/MS) system (high-performance LC (HPLC), Shim-pack UFLC SHIMADZU CBM30A system, www.shimadzu.com.cn/; MS, Applied Biosystems 6500 Q TRAP, www.appliedbiosystems.com.cn/) was used to analyze the sample extracts (HPLC: column, Waters ACQUITY UPLC HSS T3 C18 $(1.8 \mu \mathrm{m}, 2.1 \mathrm{~mm} * 100 \mathrm{~mm})$; solvent system: water $(0.04 \%$ acetic acid); acetonitrile $(0.04 \%$ acetic acid); gradient program, $95: 5 \mathrm{v} / \mathrm{v}$ at $0 \mathrm{~min}, 5: 95 \mathrm{v} / \mathrm{v}$ at $11.0 \mathrm{~min}, 5: 95 \mathrm{v} / \mathrm{v}$ at $12.0 \mathrm{~min}, 95: 5 \mathrm{v} / \mathrm{v}$ at $12.1 \mathrm{~min}, 95: 5 \mathrm{v} / \mathrm{v}$ at $15.0 \mathrm{~min}$; temperature, $40^{\circ} \mathrm{C}$; injection volume: $2 \mu \mathrm{L}$; flow rate, $0.40 \mathrm{~mL} / \mathrm{min})$. Then, the effluent was alternatively connected to an ESI-triple quadrupole-linear ion trap (Q TRAP)-MS.

A triple quadrupole-linear ion trap mass spectrometer (Q TRAP, API 6500 Q TRAP LC/MS/MS system) was used to acquire Linear ion trap (LIT) and triple quadrupole (QQQ) scans. The triple quadrupole-linear ion trap mass spectrometer was equipped with an ESI turbo ion-spray interface, operated in a positive ion mode and controlled by Analyst 1.6.3 software (AB Sciex, Boston, USA). The ESI source operation parameters were as follows: ion source, turbo spray, ion source gas I (GSI), gas II (GSII), and curtain gas (CUR) were set to 55, 60, and 25.0 psi, respectively. The collision gas (CAD) was high, ion spray voltage (IS) $5500 \mathrm{~V}$, source temperature $500{ }^{\circ} \mathrm{C}$. In QQQ and LIT modes, 10 and $100 \mu \mathrm{mol} / \mathrm{L}$ polypropylene glycol solutions were used in Instrument tuning and mass calibration, respectively. QQQ scans were acquired as multiple reaction monitoring (MRM) experiments (the collision gas (nitrogen): 5 psi). Declustering potential (DP) and collision energy (CE) for individual MRM transitions was done with further DP and CE optimization. A specific set of MRM transitions were monitored for each period according to the metabolites eluted within this period.

\subsection{Illumina Sequencing and Data Analysis}

Total RNA was extracted with an RNAprep Pure Plant Kit (Tiangen Company, Beijing, China). The quality of the total RNA was checked by electrophoresis and the concentration was determined by NanoDrop (Thermo Scientific, Wilmington, DE, USA). The cDNA libraries were created according to the standard preparation method for mRNA-seq samples (Illumina Inc, San Diego, CA, USA).

Using an Illumina HiSeq 2000 instrument (Illumina Inc., San Diego, CA, USA), the DNA library was sequenced by Novogene with three repeats. The original reading was filtered to remove ambiguous, joint and low-quality sequences before sequence assembly. After purity filtration, de novo assembly of 
the transcriptome into unigenes was carried out using Trinity, a short-read assembly program [32]. The unigene sequences were compared to protein databases (Nr, Swiss-Prot, KEGG, and Clusters of Orthologous Groups of proteins (COG)) with software Blast X (v2.2.25, National Center for Biotechnology Information, Bethesda, Maryland, USA) (e-value $<0.00001$ ). Based on the blast results, the CDS from unigene sequences were extracted and translated into peptide sequences. The blast results were also used in training ESTScan [33]. The expression levels of the unigenes were calculated based on the FPKM (fragments per kb per million reads) value. The difference in unigenes between purple and green leaf transcripts was analyzed by IDEG6 software (1.0, BGI, ShenZhen, GuangDong, China) [34]. The false discovery rate (FDR) method was introduced to determine the threshold $p$-value at FDR $\leq 0.001, \mid \log 2$ ratio $>1$ was determined by the significance of the differential expression of unigenes. All differentially abundant unigenes were mapped to the Gene ontology (GO) and KEGG databases using Blast $X$ (e-value $<0.00001$ ), and the respective numbers of unigenes for every GO and KEGG orthology $(\mathrm{KO})$ term were calculated. Based on the hypergeometric test, significantly enriched GO and $\mathrm{KO}$ terms were identified to compare these unigenes with the whole transcriptome background [35].

\subsection{Quantitative Reverse-Transcription-PCR}

The cDNA was synthesized from the same total RNA for Illumina sequencing using a reverse transcription kit (Thermo Fisher First Strand cDNA Synthesis Kit, Beijing, China). The $\beta$-ACTIN gene was selected for cDNA normalization and several differently expressed genes were selected to confirm the results of RNA-seq. The primers for the selected genes were designed by Primer 5.0 (Table S1). The qPCR was conducted with the Premix Ex Taq ${ }^{\mathrm{TM}}$ probe (TaKaRa, China) in Applied Biosystems QuantStudio3 (Thermo Fisher Company, Beijing, China). The thermal cycle for qPCR was $95^{\circ} \mathrm{C}$ for $30 \mathrm{~s}$, followed by 40 cycles of $95^{\circ} \mathrm{C}$ for $5 \mathrm{~s}$, and $60^{\circ} \mathrm{C}$ for $34 \mathrm{~s}$. The last stage was $95^{\circ} \mathrm{C}$ for $15 \mathrm{~s}, 60^{\circ} \mathrm{C}$ for $1 \mathrm{~min}$, and $95^{\circ} \mathrm{C}$ for $15 \mathrm{~s}$. Each plate was repeated three times. The 2- $\Delta \Delta \mathrm{CT}$ method was used to analyze the expression levels of the different genes [36].

\subsection{Statistical Analysis}

All statistical analyses were carried out using the software package SPSS (22.0, IBM SPSS Statistics, Chicago, Illinois, USA) for Windows 11.5 [37,38]. The method was UNIANOVA, and the POSTHOC was DUNCAN ALPHA (0.05).

\section{Conclusions}

The MYB transcription factor LrAN2 was first reported by our laboratory. It expressed only in the black fruit of Lycium ruthenicum Murry and the allele variation of LrAN2 is strictly related to the black fruit trait [23]. Overexpression of $\operatorname{LrAN2}$ activates the pathway of anthocyanin synthesis and metabolism in tobacco. Only 185 unigenes were expressed differently in leaves and 224 chemical compounds exhibited differences in accumulation. Three anthocyanins lead to the purple leaf phenotype. The main pathways of flavonoid biosynthesis were upregulated. This research provides more information about the function of MYB transcription factors in anthocyanin biosynthesis and the production of other chemical compounds. This work will help breeders to obtain new plant cultivars with high anthocyanin contents using biotechnology.

Supplementary Materials: Supplementary materials can be found at http://www.mdpi.com/1422-0067/20/20/5123/s1. Figure S1. Detection of TIC overlaps by QC sample Mass Spectrometry. Table S1. The primers used in this manuscript. Table S2. The differently-accumulation chemical compounds in leaves of transgenic lines and WT. Table S3. Summary of transcriptome sequencing data. Table S4. Distribution of the lengths of the assembled unigenes. Table S5. The annotation of assembled unigenes. Table S6. The differently expressed unigenes in leaves of transgenic lines and WT. Table S7. The relative transcript level of the unigenes relative to anthocyanin biosynthesis. Table S8. The KEGG pathways of differently expressed unigenes and differently accumulated chemical compounds 
Author Contributions: R.W. and B.L. conceived and designed the experiments. Y.Z., S.M., X.X., D.C. and Z.W. analyzed the data. D.C. provided the materials. B.L. and Y.Z. wrote the manuscript. All authors approved the submission of the manuscript

Funding: This research was financially supported by the Project of Qinghai Science and Technology Department (2018-NK-133, 2019-ZJ-982Q), West Light Talent Program of the Chinese Academy of Sciences, The Innovative Academy of Seed Design, Chinese Academy of Sciences.

Conflicts of Interest: The authors have declared that no competitive or conflicting interests exist.

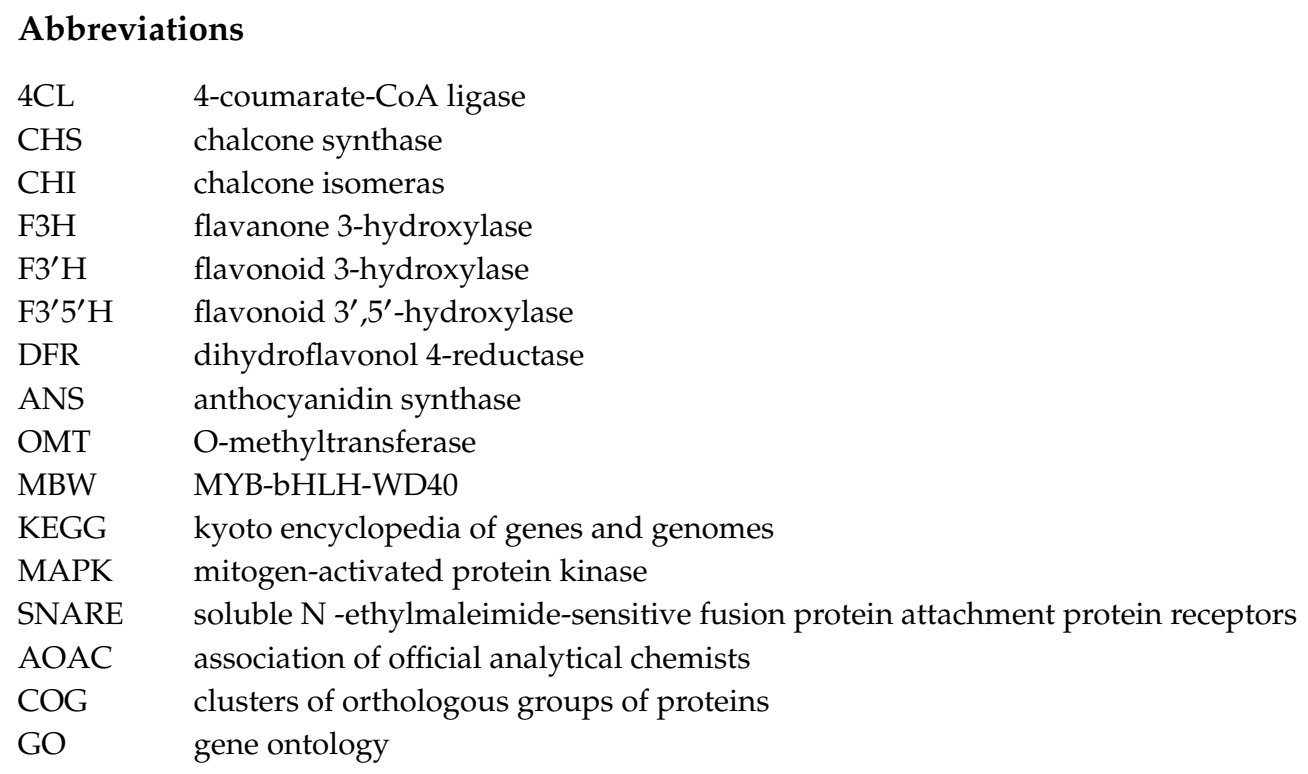

\section{References}

1. Asen, S.; Stewart, R.N.; Norris, K.H. Copigmentation of Anthocyanins in Plant-Tissues and Its Effect on Color. Phytochemistry 1972, 11, 1139-1144. [CrossRef]

2. Florenciano, E.G.; Calderon, A.A.; Munoz, R.; Barcelo, A.R. Patterns of Anthocyanin Deposition in Vacuoles of Suspension Cultured Grapevine Cells. Phyton-Int. J. Exp. Bot 1992, 53, 47-50.

3. Pojer, E.; Mattivi, F.; Johnson, D.; Stockley, C.S. The Case for Anthocyanin Consumption to Promote Human Health: A Review. Compr. Rev. Food Sci. F. 2013, 12, 483-508. [CrossRef]

4. Li, S.S.; Strid, A. Anthocyanin accumulation and changes in CHS and PR-5 gene expression in Arabidopsis thaliana after removal of the inflorescence stem (decapitation). Plant Physiol. Bioch. 2005, 43, 521-525. [CrossRef] [PubMed]

5. Honda, C.; Kotoda, N.; Wada, M.; Kondo, S.; Kobayashi, S.; Soejima, J.; Zhang, Z.L.; Tsuda, T.; Moriguchi, T. Anthocyanin biosynthetic genes are coordinately expressed during red coloration in apple skin. Plant Physiol. Bioch. 2002, 40, 955-962. [CrossRef]

6. Sun, W.; Meng, X.Y.; Liang, L.J.; Li, Y.Q.; Zhou, T.T.; Cai, X.Q.; Wang, L.; Gao, X. Overexpression of a Freesia hybrida flavonoid 3-O-glycosyltransferase gene, Fh3GT1, enhances transcription of key anthocyanin genes and accumulation of anthocyanin and flavonol in transgenic petunia (Petunia hybrida). In Vitro Cell Dev.-Pl. 2017, 53, 478-488. [CrossRef]

7. Zhu, Z.G.; Li, G.R.; Liu, L.; Zhang, Q.T.; Han, Z.; Chen, X.S.; Li, B. A R2R3-MYB Transcription Factor, VvMYBC2L2, Functions as a Transcriptional Repressor of Anthocyanin Biosynthesis in Grapevine (Vitis vinifera L.). Molecules 2019, 24, 92. [CrossRef] [PubMed]

8. Li, Y.D.; Meng, F.L.; Zheng, Y.N. Study of Anthocyanins in fruit of different Vaccinium genotypes. Acta Hortic. 2006, 715, 589-594. [CrossRef]

9. Lloyd, A.; Brockman, A.; Aguirre, L.; Campbell, A.; Bean, A.; Cantero, A.; Gonzalez, A. Advances in the MYB-bHLH-WD Repeat (MBW) Pigment Regulatory Model: Addition of a WRKY Factor and Co-option of an Anthocyanin MYB for Betalain Regulation. Plant Cell Physiol. 2017, 58, 1431-1441. [CrossRef]

10. Chiu, L.W.; Zhou, X.; Burke, S.; Wu, X.; Prior, R.L.; Li, L. The purple cauliflower arises from activation of a MYB transcription factor. Plant Physiol. 2010, 154, 1470-1480. [CrossRef] 
11. Espley, R.V.; Hellens, R.P.; Putterill, J.; Stevenson, D.E.; Kutty-Amma, S.; Allan, A.C. Red colouration in apple fruit is due to the activity of the MYB transcription factor, MdMYB10. Plant J. 2007, 49, 414-427. [CrossRef] [PubMed]

12. Kim, C.Y.; Ahn, Y.O.; Kim, S.H.; Kim, Y.H.; Lee, H.S.; Catanach, A.S.; Jacobs, J.M.; Conner, A.J.; Kwak, S.S. The sweet potato IbMYB1 gene as a potential visible marker for sweet potato intragenic vector system. Physiol. Plant 2010, 139, 229-240. [PubMed]

13. Yamagishi, M.; Shimoyamada, Y.; Nakatsuka, T.; Masuda, K. Two R2R3-MYB genes, homologs of Petunia AN2, regulate anthocyanin biosyntheses in flower Tepals, tepal spots and leaves of asiatic hybrid lily. Plant Cell Physiol. 2010, 51, 463-474. [CrossRef] [PubMed]

14. Pattanaik, S.; Kong, Q.; Zaitlin, D.; Werkman, J.R.; Xie, C.H.; Patra, B.; Yuan, L. Isolation and functional characterization of a floral tissue-specific R2R3 MYB regulator from tobacco. Planta 2010, 231, 1061-1076. [CrossRef]

15. Cho, J.S.; Nguyen, V.P.; Jeon, H.W.; Kim, M.H.; Eom, S.H.; Lim, Y.J.; Kim, W.C.; Park, E.J.; Choi, Y.I.; Ko, J.H. Overexpression of PtrMYB119, a R2R3-MYB transcription factor from Populus trichocarpa, promotes anthocyanin production in hybrid poplar. Tree Physiol. 2016, 36, 1162. [CrossRef]

16. Li, Y.; Tu, K.; Zheng, S.; Wang, J.; Li, Y.; Hao, P.; Li, X. Association of feature gene expression with structural fingerprints of chemical compounds. J. Bioinform. Comput. Biol. 2011, 9, 503-519. [CrossRef]

17. Fujii, K.; Murase, T.; Beppu, S.; Saida, K.; Takino, H.; Masaki, A.; Ijichi, K.; Kusafuka, K.; Iida, Y.; Onitsuka, T.; et al. MYB, MYBL1, MYBL2 and NFIB gene alterations and MYC overexpression in salivary gland adenoid cystic carcinoma. Histopathology 2017, 71, 823-834. [CrossRef]

18. Janssen, B.J.; Lund, L.; Sinha, N. Overexpression of a homeobox gene, LeT6, reveals indeterminate features in the tomato compound leaf. Plant Physiol. 1998, 117, 771-786. [CrossRef]

19. Wei, C.; Liang, G.; Guo, Z.; Wang, W.; Zhang, H.; Liu, X.; Yu, S.; Xiong, L.; Jie, L. A Novel Integrated Method for Large-Scale Detection, Identification, and Quantification of Widely Targeted Metabolites: Application in the Study of Rice Metabolomics. Mol. Plant 2013, 6, 1769-1780.

20. Zhang, N.; Liu, B.; Ma, C.; Zhang, G.; Chang, J.; Si, H.; Wang, D. Transcriptome characterization and sequencing-based identification of drought-responsive genes in potato. Mol. Biol. Rep. 2014, 41, 505-517. [CrossRef]

21. Dorn, K.M.; Fankhauser, J.D.; Wyse, D.L.; Marks, M.D. De novo assembly of the pennycress (Thlaspi arvense) transcriptome provides tools for the development of a winter cover crop and biodiesel feedstock. Plant J. 2013, 75, 1028-1038. [CrossRef] [PubMed]

22. Firon, N.; LaBonte, D.; Villordon, A.; Kfir, Y.; Solis, J.; Lapis, E.; Perlman, T.S.; Doron-Faigenboim, A.; Hetzroni, A.; Althan, L. Transcriptional profiling of sweetpotato (Ipomoea batatas) roots indicates down-regulation of lignin biosynthesis and up-regulation of starch biosynthesis at an early stage of storage root formation. BMC Genomics 2013, 14, 460. [CrossRef] [PubMed]

23. Zong, Y.; Zhu, X.; Liu, Z.; Xi, X.; Li, G.; Cao, D.; Wei, L.; Li, J.; Liu, B. Functional MYB transcription factor encoding gene AN2 is associated with anthocyanin biosynthesis in Lycium ruthenicum Murray. BMC Plant Biol. 2019, 19, 169. [CrossRef] [PubMed]

24. Christopher Dean, G.; Paula, C.; Virginia, W. A multidrug resistance-associated protein involved in anthocyanin transport in Zea mays. Plant Cell 2004, 16, 1812-1826.

25. De Vetten, N.; Ter Horst, J.; Van Schaik, H.P.; De Boer, A.; Mol, J.; Koes, R. A cytochrome b5 is required for full activity of flavonoid 3', 5'-hydroxylase, a cytochrome P450 involved in the formation of blue flower colors. Proc. Natl. Acad. Sci. USA 1999, 96, 778-783. [CrossRef]

26. Vincenzo, D.A.; Riccardo, A.; Giorgia, B.; Immacolata, C.; Mar, C.M.; Ana Beatriz, C.S.; Pasquale, C.; Carlo, F.; Francesca, P.; Domenico, C. High AN1 variability and interaction with basic helix-loop-helix co-factors related to anthocyanin biosynthesis in potato leaves. Plant J. 2015, 80, 527-540.

27. Blank, M.L.; Lee, T.; Fitzgerald, V.; Snyder, F. A specific acetylhydrolase for 1-alkyl-2-acetyl-snglycero-3-phosphocholine (a hypotensive and platelet-activating lipid). J. Biol. Chem. 1981, 256, 175-178.

28. Li, X.; Hao, F.; Hu, X.; Wang, H.; Dai, B.; Wang, X.; Liang, H.; Cang, M.; Liu, D. Generation of Tbeta4 knock-in Cashmere goat using CRISPR/Cas9. Int. J. Biol. Sci. 2019, 15, 1743-1754. [CrossRef]

29. Zhu, Q.; Sui, S.; Lei, X.; Yang, Z.; Lu, K.; Liu, G.; Liu, Y.G.; Li, M. Ectopic Expression of the Coleus R2R3 MYB-Type Proanthocyanidin Regulator Gene SsMYB3 Alters the Flower Color in Transgenic Tobacco. PLoS ONE 2015, 10, e0139392. [CrossRef] 
30. Zhu, H.-F.; Fitzsimmons, K.; Khandelwal, A.; Kranz, R.G. CPC, a single-repeat R3 MYB, is a negative regulator of anthocyanin biosynthesis in Arabidopsis. Mol. Plant 2009, 2, 790-802.

31. Horsch, R.B.; Fry, J.E.; Hoffmann, N.L.; Eichholtz, D.; Rogers, S.G.; Fraley, R.T. A Simple and General Method for Transferring Genes into Plants. Science 1985, 227, 1229-1231.

32. Cabau, C.; Escudie, F.; Djari, A.; Guiguen, Y.; Bobe, J.; Klopp, C. Compacting and correcting Trinity and Oases RNA-Seq de novo assemblies. PeerJ 2017, 5. [CrossRef] [PubMed]

33. Iseli, C.; Jongeneel, C.V.; Bucher, P. ESTScan: A program for detecting, evaluating, and reconstructing potential coding regions in EST sequences. Proc. Int. Conf. Intell. Syst. Mol. Biol. 1999, 1999, 138-148.

34. Romualdi, C.; Bortoluzzi, S.; D'Alessi, F.; Danieli, G.A. IDEG6: A web tool for detection of differentially expressed genes in multiple tag sampling experiments. Physiol. Genomics 2003, 12, 159-162. [CrossRef]

35. Rivals, I.; Personnaz, L.; Taing, L.; Potier, M.-C. Enrichment or depletion of a GO category within a class of genes: Which test? Bioinformatics 2007, 23, 401-407. [CrossRef]

36. Hurley, J.H.; Dasgupta, A.; Andrews, P.; Crowell, A.M.; Ringelberg, C.; Loros, J.J.; Dunlap, J.C. A Tool Set for the Genome-Wide Analysis of Neurospora crassa by RT-PCR. G3 (Bethesda) 2015, 5, 2043-2049. [CrossRef]

37. An, S.L.; Mo, Y.X.; Ou, C.Q. [Probit analysis with SPSS 10.0 software]. Di Yi Jun Yi Da Xue Xue Bao 2002, 22, 1019-1021.

38. An, S.; Zhang, Y.; Chen, Z. [Analysis of binary classification repeated measurement data with GEE and GLMMs using SPSS software]. Nan Fang Yi Ke Da Xue Xue Bao 2012, 32, 1777-1780.

(C) 2019 by the authors. Licensee MDPI, Basel, Switzerland. This article is an open access article distributed under the terms and conditions of the Creative Commons Attribution (CC BY) license (http://creativecommons.org/licenses/by/4.0/). 